\title{
Pedicle Lesion in the Toddler: A Radiologic Review
}

\author{
Rajeev K. Patel, MD*, Paul T. Rubery Jr MD*, Steven Myers MD**, and Clifford R. Everett MD*
}

\section{Case Presentation}

E.H. is a 5 year-old girl who presented to an academic multidisciplinary spine center with a chief complaint of abdominal and back pain for one year. The patients' pain, which progressed from intermittent to constant, was located on the right side of the peri-umbilical and thoracolumbar areas. She reported incomplete relief with ibuprofen and codeine. She also reported generalized malaise, constipation, and insomnia due to night pain. There was no weight loss or fever reported. The patients' musculoskeletal and abdominal exam was normal except for midline lower thoracic pain to palpation. She underwent a complete gastrointestinal and genito-urinary evaluation including computed tomography (CT) of the abdomen and pelvis, renal ultrasound, and urinalysis none of which demonstrated significant pathology. In addition, complete hematology was within normal limits with the exception of an elevated erythrocyte sedimentation rate of 40. Plain films, bone scan, CT with sagittal and coronal reformations and magnetic resonance imaging (MRI) of the thoracic spine were ordered.

\section{DisCUSSION}

Plain films of the thoracic spine demonstrated an osteosclerotic lesion of

From University of Rochester Spine Center, *The Dept. of Orthopaedic Surgery University of Rochester School of Medicine, Strong Memorial Health System, Rochester, NY 14642 and ${ }^{\star *}$ The Dept. of Radiology, University of Rochester School of Medicine, Strong Memorial Health System, Rochester, NY 14642. Address Correspondence: Rajeev K. Patel, MD, University of Rochester Spine Center, 601 Elmwood Avenue, Box\#665, Rochester, NY 14642. Email: Rajeev_Patel@urmc.rochester.edu

There has been no support in the form of grants, equipment, or drugs. the left T10 pedicle (Fig 1). The remainder of the plain films were normal. Bone Scan with Single-Photon Computed Tomography (SPECT) imaging demonstrated a focal abnormality of the T10 vertebral pedicle. Thoracic spine CT with sagittal and coronal reformations demonstrated an expansile, lytic lesion of the T10 pedicle measuring $0.9 \times 0.9 \mathrm{~cm}$ with destruction of the cortex medially and associated sclerosis of the vertebral body (Fig 2A-C). There was calcification present within the lesion. No neural compression was identified. MRI of the thoracic spine with and without Gadolinium demonstrated a $1.2 \times 0.8 \times 0.7 \mathrm{~cm}$ lesion of the left side of the T10 pedicle and posterolateral body with edema in the adjacent posterior elements including the lamina and superior articular process (Fig 3A-F). There is also enhancement through this region and into the adjacent paraspinal tissues. There was no compromise of the spinal canal with normal signal within the spinal cord. The lesion was low to intermediate signal on $\mathrm{T} 1$ and heterogeneous to slightly increased signal on T2. Potential etiologies included osteoblastoma, osteoid osteomas, giant cell tumor, aneurysmal bone cyst, metastasis (most commonly from Ewing's sarcoma) and eosinophil-

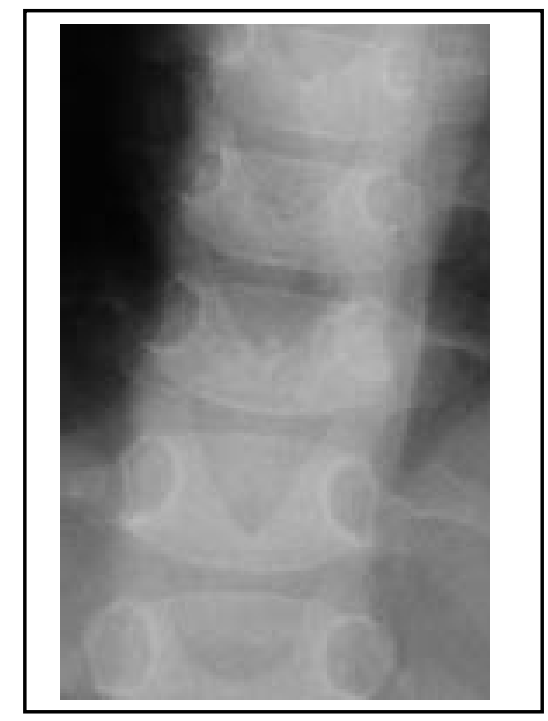

Fig 1. Plain film of thoracic spine demonstrating osteosclerotic lesion of the left T10 pedicle

ic granuloma.

Common modalities used in imaging spinal tumors include plain radiography, CT, MRI, and nuclear scintigrapy/ bone scan with SPECT. Plain radiography is a readily available low cost survey of spinal bone. The problem remains a lack of sensitivity for early lesions and lack of vi-

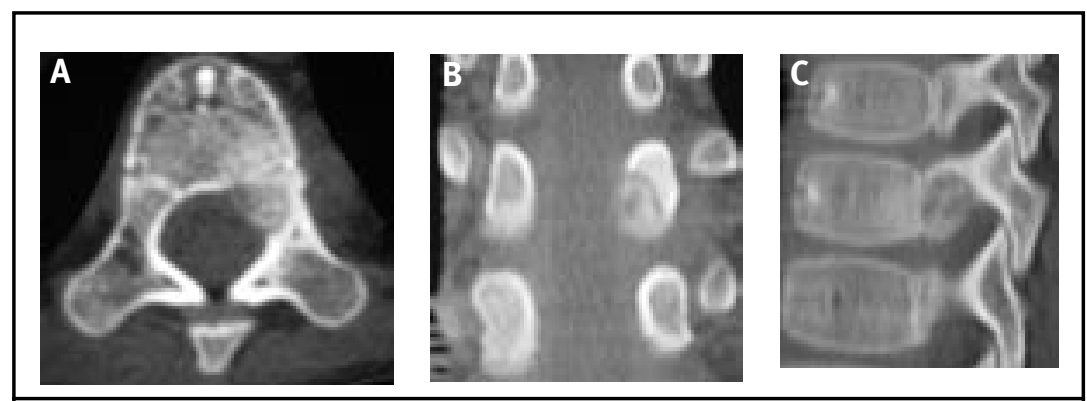

Fig 2. Thoracic CT scan demonstrating an expansile, lytic lesion of the T10 pedicle with destruction of the cortex medially. 

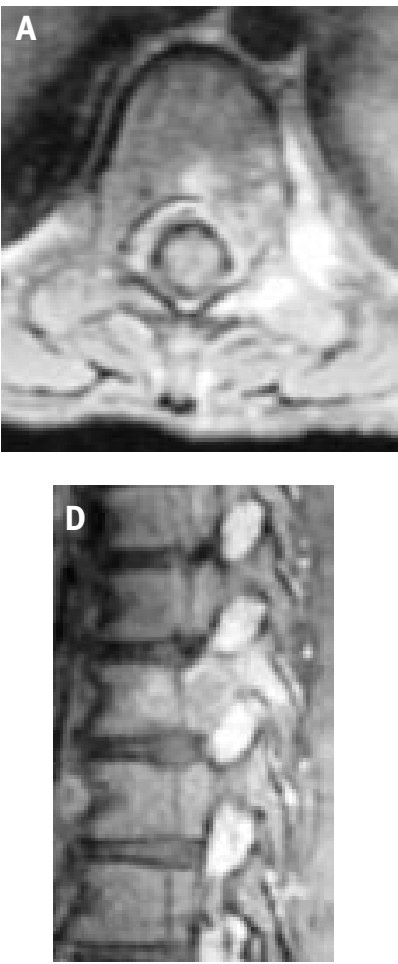
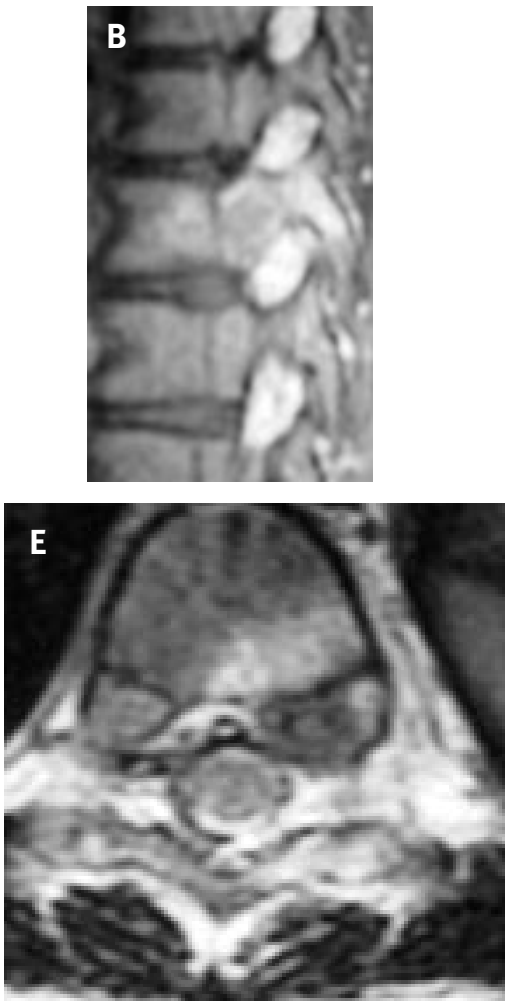
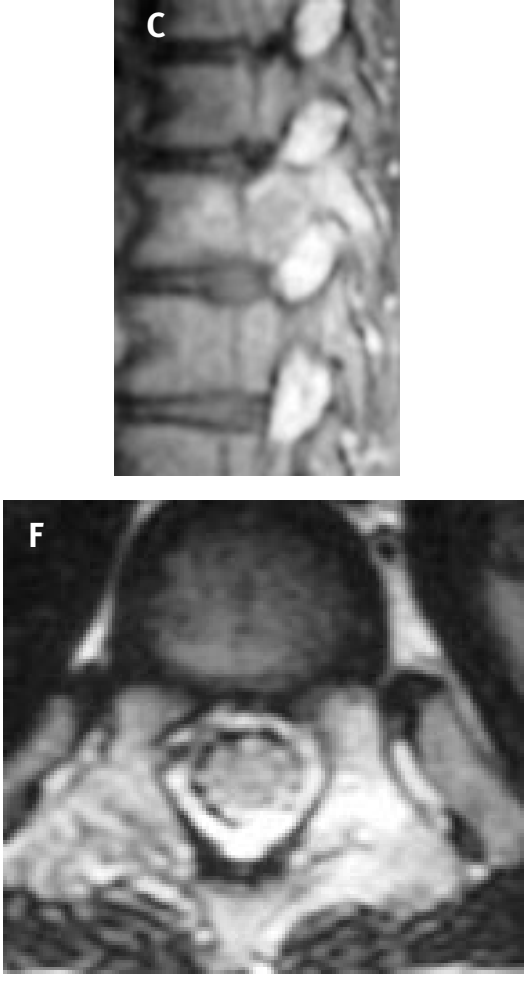

Fig 3. MRI of thoracic spine with and without demonstrating lesion of the T10 pedicle with edema in the adjacent posterial elements

sualization of intraspinal lesions. CT is helpful in determining the lytic or blastic nature of a lesion and soft tissue calcification. It has limited sensitivity for marrow replacement processes and intradural processes. Scintigraphy is helpful in evaluating the entire skeletal system for metastatic spread and possibly finding an alternative site for biopsy than the spine, however, it is limited in anatomical detail. Scintigraphy also has difficulty in disease processes without bone repair such as in multiple myeloma. MRI is the imaging modality that is ideally suited for marrow replacement and intradural tumors. It is also helpful in ruling out other disorders such as hemangioma or infection.

Osteoblastoma is a rare, benign primary tumor of bone accounting for approximately $1 \%$ of all primary bone tumors and $3 \%$ of all benign bone tumors (1). Although they are described in almost every bone, 7 to $25 \%$ of all osteoblastomas are located in the lumbar spine, $66 \%$ in the neural arch, $24 \%$ in the posterior elements and only $3 \%$ in the vertebral body $(2,3)$. Pain and local tenderness may appear before the lesion becomes evi- dent on plain films. The usual delay to diagnosis varies between 9 and 12 months $(1,2)$. Osteoblastomas are composed of an abundantly vascular connective-tissue stroma with active production of osteoid and primitive woven bone. A central lucency with a variable zone of peripheral sclerosis is the typical pattern; osteolysis and mass effect are frequently present $(2,4)$. A soft-tissue epidural extension with well-defined margins and a lucent or ossified center or a dense sclerotic reaction can accompany the lesion (4). Diffuse, reactive edema and nonspecific inflammatory infiltrate in the paraspinous soft tissue has been described (5). Periosteal origin is rare, but cortical destruction, which may result in neural compression, is reported in more than $50 \%$ of the cases (2). Plain films and CT show the expansile lytic or osteosclerotic lesion with thinned surrounding cortex. A tumor confined to a vertebral body is quite unusual as there is usually posterior element involvement (3). Descriptions of osteoblastoma on MRI can be variable. Bissel et al (6) described a sacral osteoblastoma which had a homogenously isointense sig- nal on T1-weighted images. Syklawer et al (7) reported on cases of osteoblastoma with intermediate signal intensity of T1weighted images and progressively decreasing signal with T2-weighting. Kroon et al (8) reported cases of osteoblastoma demonstrating low and intermediate signal intensity on T1-weighted images and predominantly high signal intensity on T2-weighted images.

Osteoid osteomas account for $6 \%$ of benign spine tumors (9). Only $10 \%$ of these tumors occur within the axial skeleton (10). They are often diagnosed in the second decade of life. There is a male predilection and patients present with pan that is worse at night and relieved by salicylates and non-steroidal anti-inflammatory agents. Osteoid osteomas are found within the posterior elements of the lumbar spine (59\% of cases) and cervical spine (27\% of cases) (10). Imaging studies are directed at demonstrating a small nidus which consists of fibrovascular stroma surrounded by sclerotic osseous reaction. Plain radiographs show a round radiolucent nidus that is surrounded by sclerotic bone. A scoliotic spine is frequently ob- 
served. CT confirms the presence of a hypodense 1.5 to $2 \mathrm{~cm}$ nidus that may contain central calcification. CT is probably the optimal modality for evaluating these lesions (10). Skeletal scintigraphy is also useful in detecting these lesions as they demonstrate focal increased radiotracer uptake. The MR imaging findings are less consistent, but the nidus tends to be hypo to isointense on T1-weighted images and iso- to hyperintense on T2-weighted images (10). Edema in the adjacent marrow will be hypointense on T1-weighted images and hyperintense on T2-weighted images, while the sclerosis is hypointense on these sequences. Both osteoblastoma and osteoid osteoma present similarly radiologically and are high in the differential of this patient. Therefore, as with this case, histology is generally required to make a definitive diagnosis.

Giant-cell tumors account for up to $7 \%$ of primary bone tumors of which only $7 \%$ have spinal involvement. It is more common in females in their twenties to forties (11). It involves the sacrum or vertebral body to a greater extent than the posterior elements. Plain films and CT show an expansile, lytic lesion which is capable of crossing the disc space. Bone scan has a photopenic center surrounded by increased tracer uptake. There may be hemorrhagic or necrotic changes within the lesion visible on CT. The lesion is best imaged with MRI, which shows a hypoto isointense lesion on T1 and T2 imaging. MRI also demonstrates the soft tissue component, which is a multi-compartmented heterogeneous mass. This patient did not have significant soft tissue expansion and it would be an unusual tumor within this age group.

Aneurysmal bone cysts occur before 20 years of age but usually after 5 years of age. They occur more commonly in females and within the thoracic spine but can affect long tubular bones. They are also felt by some to be a result of prior trauma (10). In other cases a pre-existing lesion may progress to the appearance of an aneurysmal bone cyst (12). The most common lesion to produce this appearance late in its course would be a giant cell tumor, which is described later in this review. Symptomatology usually corresponds to spinal cord or nerve root encroachment. The posterior elements are most frequently affected but it can effect the vertebral body or adjacent vertebrae. Plain films show an expansile lytic lesion with thinning cortical margins. CT scans also show a lytic lesion but also fluid levels or lobulated masses in some of the cases due to hemorrhage. MRI is helpful in identifying the cystic nature of these lesions with increased signal on both $\mathrm{T} 1$ and T2-weighted imaging reflecting subacute hemorrhage (13). This patient had an expansile lytic lesion on plain film and CT this was strongly considered in our initial differential diagnosis. The MRI of our patient, however, was without a primary vascular or lobulated lesion making aneurysmal bone cyst a much less likely diagnosis.

Ewings sarcoma is the most common malignant tumor of the spine in children excluding the lymphoproliferative tumors (9). Spinal involvement usually occurs due to metastatic spread. Primary spinal Ewing's sarcoma is quite uncommon accounting for only $3.5 \%$ of those with Ewing's (14). Constitutional symptoms of weight loss, fatigue, and fever are common. The vertebral body is usually involved with plain film and CT scan having a variable presentation with lytic, sclerotic, or mixed patterns visible with prominent soft tissue mass. The destructive lesion contains dense mineralized matrix. On MRI this mineralization is hypointense on all sequences. For the lesion in general the tumor is hypo- to isointense on T1-weighted images and iso-to hyperintense on T2-weighted images. This patient demonstrated minimal soft tissue expansion and the pedicle was involved to a greater extent than the vertebral body.

Eosinophilic granuloma produces destruction of bone. The skull is involved greater than the spine but vertebral lesions have been reported in $10-15 \%$ of cases (14). It typically produces vertebral collapse with local pain and spasm. Due to resultant kyphosis or local tumor compression, neurologic complaints can occur. Plain films show a lytic lesion without clear margins.

This patient underwent a transpedicular removal of tumor from the left side of T10 using image-guided stereotactic navigation. The tissue diagnosis was consistent with osteoblastoma. Post-operative films demonstrate no vertebral collapse and a lytic area at the surgical site with minimal sclerosis. Postoperatively she had $90 \%$ relief of her symptoms with only mild incisional pain. She did not require any adjuvant therapy. She was able to resume most of her usual activities only occasionally utilizing ibuprofen or acetaminophen for pain control. She no longer has night pain.

\section{Author Affiliation: \\ Rajeev K. Patel, MD}

Assistant Professor, Department of Orthopaedic Surgery, University of Rochester School of Medicine, Strong Memorial Health System, Rochester, NY 14642. or E-mail: Rajeev_Patel@urmc.rochester.edu

\section{Paul T. Rubery Jr, MD}

Associate Professor, Department of Orthopaedic Surgery, University of Rochester School of Medicine, Strong Memorial Health System, Rochester, NY 14642. or E-mail: paul_rubery@urmc.rochester.edu

\section{Steven Myers, MD}

Department of Radiology, University of Rochester School of Medicine, Strong Memorial Health System, Rochester, NY 14642 or E-mail: Steven_Myers@urmc.rochester.edu , and

\section{Clifford R. Everett, MD}

Assistant Professor, Department of Orthopaedic Surgery, University of Rochester School of Medicine, Strong Memorial Health System, Rochester, NY 14642 or E-mail: Clifford_Everett@urmc.rochester. edu

\section{REFERENCES}

1 Dahlin DC, Unni KK. Bone tumors: general aspects and data on 8,542 cases. $4^{\text {th }}$ ed. Charles C. Thomas, Springfield, 1986, pp 102-118.

2 McLeod RA, Dahlin DC, Beaubout JW. The spectrum of osteoblastoma. Am / Rad 1976; 126: 324-335.

3 Nemoto O, Moser RP, Van Dam BE et al. Osteoblastoma of the spine. Spine 1990; 15: $1272-1280$.

4 Seki T, Fukuda H, Ishii Y. Malignant transformation of a benign osteoblastoma: a case report. J Bone J Surg 1975; 57: 424427.

5 Crim JR, Mirra JM, Eckarolt JJ et al. Widespread inflammation response to osteoblastoma: the flare phenomenon. Radiology 1990; 177: 835-836.

6 Bissell GS, Kaufman RA, Tawbin R et al. Case Report 452: recurrent sacral osteoblastoma. Skel Radiol 1987; 16: 666-669.

7 Syklawer R, Osborn RE, Kerber CW et al. 
Magnetic resonance imaging of vertebral osteoblastoma: a report of two cases. Surg Neurol 1990; 34: 421-426.

8 Kroon HM, Schurman J. Osteoblastoma: clinical and radiologic findings in 98 new cases. Radiology 1990; 175: 783-790.

9 Osborn AG. Tumors, cysts and tumorlike lesions of the spine and spinal cord. In Osborn AG (ed): Diagnostic Neuroradiology. Mosby, St. Louis, 1994, pp. 876-919.
Murphey MD, Andrews CL, Fleming DJ et al. Primary tumors of the spine: Radiologic-pathologic correlation. RadioGraphics 1996; 16:1131-1158.

11 Bazan C: Imaging of lumbosacral spine neoplasms. Neuroimaging Clin North Am 1993; 3:591-608.

12 Bonakdarpour A, Levy WM, Aegerter E. Primary and secondary aneurysmal bone cyst: a radiological study of 75 cases. $\mathrm{Ra}$ - diology 1978; 126: 75-83.

13 Kransdorf JJ, Sweet DE. Aneurysmal bone cyst: Concept, controversy, clinical presentation, and imaging. Am J Roentgenol 1995; 164:573- 580 .

14 Weinstein JN, McLain RF. Tumors of the spine. In Rothman RH, Simeone FA (eds): The Spine. W.B. Saunders, Philadelphia, 1992, pp 1279-1318. 